\title{
Лазерная трансnynuллярная mермотераnuя ретuнобластомы
}

\author{
А.А. Яровойㅁ, Д.П. Володин ${ }^{1}$, В.А. Яровая ${ }^{1}$, Т.Л. Ушакова ${ }^{2,3}$, Е.С. Котова ${ }^{1}$, А.М. Чочаева ${ }^{1}$, В.Г. Поляков ${ }^{2-4}$ \\ ${ }^{\prime}$ ФГАУ «Национальный медицинский исследовательский центр «Межкотраслевой научно-технический комплекс «Микро- \\ хирургия глаза» имени академика С.Н. Федорова» Минздрава России; Россия, 127486, Москва, Бескудниковский бул., 59а; \\ ${ }^{2}$ ФГБУ «НМИЦ онкологии им. Н.Н. Блохина» Минздрава России; Россия, 115478, Москва, Каширское шоссе, 23; \\ ${ }^{3}$ ФГБОУ ДПО «Российская медицинская академия непрерывного профессионального образования» Минздрава России; \\ Россия, 125993, Москва, ул. Баррикадная, 2/1, стр. 1; ${ }^{4}$ ФГАОУ ВО «Российский национальный исследовательский \\ медицинский университет имени Н.И. Пирогова» Минздрава России; Россия, 117997, Москва, ул. Островитянова, 1
}

Контактные данные: Денис Павлович Володин volodin.den2016@yandex.ru

Введение. Транспупиллярная термотерапия (ТТТ) является одним из основных методов разрушения малых ретинобластом (РБ), при этом количество исследований в литературе, посвященных данной проблеме, невелико, и они представлены отдельными аспектами применения данного метода.

Цель исследования - оценить эффективность ТТТ при лечении детей с РБ.

Материалы и методы. В период с 2011 по 2020 г. методом ТТТ пролечены 177 детей (224 глаза, 1156 очагов) с РБ. Из них 99 (56 \%) пациентов были мальчики, 78 (44 \%) - девочки. Средний возраст на момент лечения составил 16,8 месяца (от 0 до 86 месяцев). Бинокулярная форма РБ наблюдалась у 128 (72,3 \%) пациентов, монокулярная - у 49 (27,7\%). В 51 (28,8 \%) случае ТTТ проведена на единственном глазу. ТТТ выполнялась на глазах, имевщих РБ групn $A(n=43 ; 19 \%), B(n=81 ; 36 \%)$, $C(n=31 ; 14 \%), D(n=63 ; 28 \%), E(n=6 ; 3 \%)$. Всего методом ТТТ пролечены 1156 очагов. Постэкваториально локализовались 488 (42\%) очагов (из них 27 были расположены юкстапапиллярно, 23 - в макулярной зоне, 22 - парамакулярно). Преэкваториальную локализацию (на средней и крайней периферии глазного дна) имели 668(58\%) очагов. Количество очагов в 1 глазу варьировало от 1 до 48 (в среднем - 5). Средняя толщина опухоли составила 1,1 мм (от 0,2 до 4,5 мм), средняя протяженность - 2,2 мм (от 0,3 до 13,4 мм). ТТТ проводилась с использованием диодного лазера со следующими параметрами: длина волны - 810 нм, диаметр пятна - 1200 мкм, мощность от 200 до 800 мВт (средняя - 350 м Вт), экспозиция - от 3 до 15 с при аппликационном режиме и непрерывная - при сканирующем режиме.

Результаты. Полная регрессия опухоли после проведения ТТТ была достигнута в 92 \% случаев (1064 очага). В 0,7\% (8 очагов) наблюдалась частичная регрессия опухоли со стабилизацией. Среднее количество сеансов ТТТ для достижения полной регрессии составило 1,7 (от 1 до 10). Полная регрессия опухоли после 1 сеанса ТТТ наблюдалась в 54 \% случаев (622 очага), после 2 сеансов - в $11 \%$ (132 очага), после 3 сеансов - в $7 \%$ (85 очагов), после 4 сеансов и более - в $19 \%$ (225 очагов). В 7 \% наблюдений (82 очага) в связи с прогрессией опухоли потребовалось применение других методов лечения (брахитерапия, криодеструкция, стереотаксическая радиохирургия). Были сохранены 209 (93 \%) глаз. В связи с продолженным ростом опухоли, тотальной отслойкой сетчатки, гемофтальмом или субатрофией глазного яблока были энуклеированы 15 (7\%) глаз. Средний срок наблюдения после ТТТ составил 35,5 мес (от 3 до 112 мес).

Заключение. ТТТ является высокоэффективным методом лечения РБ и может применяться для разрушения первичных опухолей малого размера как постэкваториальной, так и преэкваториальной локализации, остаточной опухоли после неэффективности других методов локального лечения. Также ТТТ обладает эффективностью в лечении больших очагов кавитарной РБ, расположенных в функционально значимых зонах глазного дна.

Ключевые слова: ретинобластома, транспупиллянная термотерапия, офтальмоонкология, локальное лечение

Для цитирования: Яровой А.А., Володин Д.П., Яровая В.А., Ушакова Т.Л., Котова Е.С., Чочаева А.М., Поляков В.Г. Лазерная транспупилляная термотерапия ретинобластомы. Российский журнал детской гематологии и онкологии 2021;8(3):43-9.

\section{Transpupillary laser thermotherapy of retinoblastoma}

A.A. Yarovoy ${ }^{1}$, D.P. Volodin', V.A. Yarovaya ${ }^{1}$, T.L. Ushakova ${ }^{2,3}$, E.S. Kotova ${ }^{1}$, A.M. Chochaeva ${ }^{1}$, V.G. Polyakov ${ }^{2-4}$

${ }^{1}$ National Medical Research Center Academician S.N. Fyodorov Intersectoral Scientific and Technical Complex "Eye microsurgery”,

Ministry of Health of Russia; 59 a Beskudnikovsky Blvd., Moscow, 127486, Russia; ${ }^{2}$ Research Institute of Pediatric Oncology and Hematology of N.N. Blokhin National Medical Research Centre of Oncology, Ministry of Health of Russia; 23 Kashirskoe Shosse, Moscow, 115478, Russia; ${ }^{3}$ Russian Medical Academy of Continuous Professional Education, Ministry of Health of Russia; Bld. 1, 2/1 Barrikadnaya St., Moscow, 125993, Russia; ${ }^{4}$ N.I. Pirogov Russian National Research Medical University, Ministry of Health of Russia; 1 Ostrovityanova St., Moscow, 117997, Russia

Introduction. Despite the fact that transpupillary thermotherapy (TTT) is one of the main local methods of small retinoblastoma (RB) destruction, only a few studies have been published on the use of this method, and they are devoted only to certain aspects of the application of TTT. Purpose of the study - to evaluate the effectiveness of TTT in the treatment of children with RB.

Material and methods. In the period from 2011 to 2020, 177 children (224 eyes, 1156 tumors) with RB were treated by TTT. Of these, 99 (56 \%) patients were boys, 78 (44\%) - girls. The mean age at the time of treatment was 16.8 months (from 0 to 86 months). Bilateral 
RB was observed in $128(72.3 \%)$ patients, monolateral - in $49(27.7 \%)$. In 51 (28.8\%) cases, TTT was performed on an only eye. TTT was performed on eyes that had RB of groups $A(n=43 ; 19 \%), B(n=81 ; 36 \%), C(n=31 ; 14 \%), D(n=63 ; 28 \%), E(n=6 ; 3 \%)$. In total, 1156 tumors were treated by TTT. 488 (42\%) tumors were localized post-equatorially (of which 27 were located juxtapapillary, 23 - in the macular zone, 22 - paramacular). 668 (58\%) foci had pre-equatorial localization (on the middle and far periphery of the fundus). The number of foci in one eye varied from 1 to 48 (mean - 5). The mean tumor thickness was $1.1 \mathrm{~mm}$ (from 0.2 to 4.5 ), the mean base diameter was $2.2 \mathrm{~mm}$ (from 0.3 to 13.4). TTT was performed using a diode laser with the following parameters: wavelength $810 \mathrm{~nm}$, spot diameter - 1200 microns, power from 200 to $800 \mathrm{~mW}$ (mean - $350 \mathrm{~mW}$ ), exposure-from 3 to $15 \mathrm{~s}$ in the application mode, and continuous in the scanning mode.

Results. Complete tumor regression after TTT was achieved in $92 \%$ of cases (1064 tumors). Incomplete regression of the tumor with stabilization was achieved in $0.7 \%$ (8 tumors). The average number of TTT sessions to achieve full regression was 1.7 (from 1 to 10 ). Complete tumor regression after 1 TTT session was achieved in $54 \%$ of cases (622 tumors), after 2 sessions - in $11 \%$ (132 tumors), after 3 sessions - in $7 \%$ (85 tumors), after 4 or more sessions - in $19 \%$ (225 tumors). In $7 \%$ of cases (82 tumors), due to the progression of the tumor, other treatment methods (brachytherapy, cryotherapy, stereotactic radiosurgery) were applied. 209(93\%) eyes were preserved. 15 (7\%) eyes were enucleated due to continued tumor growth, total retinal detachment, vitreous hemorrhage, or subatrophy of the eyeball. The mean follow-up after TTT was 35.5 months (from 3 to 112 months).

Conclusion. TTT is a highly effective method of RB treatment and can be used for destruction of small primary foci of both post-equatorial and pre-equatorial localization, residual tumors after inefficiency of other local methods. TTT is also effective in the treatment of large cavitary tumors located in functionally significant areas of the retina.

Key words: retinoblastoma, transpupillary thermotherapy, ocular oncology, local treatment

For citation: Yarovoy A.A., Volodin D.P., Yarovaya V.A., Ushakova T.L., Kotova E.S., Chochaeva A.M., Polyakov V.G. Transpupillary laser thermotherapy of retinoblastoma. Russian Journal of Pediatric Hematology and Oncology 2021;8(3):43-9.

\section{Информация об авторах}

А.А. Яровой:д.м.н., заведующий отделом офтальмоонкологии и радиологии НМИЦ «МНТК «Микрохирургия глаза» им. акад. С.Н. Федорова, e-mail: yarovoyaa@yandex.ru; https://orcid.org/0000-0003-2219-7054, SPIN-код: 9401-4489

Д.П.Володин: врач-ординаторотделения офтальмоонкологиии радиологии НМИЦ «МНТК Микрохирургия глаза» им. акад. С.Н. Федорова, e-mail: volodin.den2016@yandex.ru; https://orcid.org/0000-0002-3660-7803, SPIN-код: 7404-9620

В.А. Яровая: врач-офтальмолог отдела офтальмоонкологии и радиологии НМИЦ «МНТК «Микрохирургия глаза» им. акад. С.Н. Федорова, e-mail: verandreevna@gmail.com; https://orcid.org/0000-0001-8937-7450, SPIN-код: 4000-0180

Т.Л. Ушакова: д.м.н., и.о. заведующего детским онкологическим отделением хирургических методов лечения с проведением химиотерапии № 1 (опухолей головы и шеи) НИИ детской онкологии и гематологии НМИЦ онкологии им. Н.Н. Блохина, профессор кафедры детской онкологии им. акад. Л.А. Дурнова РМАНПО, e-mail: ushtat07@mail.ru; https://orcid.org/0000-0003-3263-6655, SPIN-код: 2065-8779

Е.С. Котова: врач-аспирант отделения офтальмоонкологии и радиологии НМИЦ «МНТК «Микрохирургия глаза» им. акад. С.Н. Федорова, e-mail: elenkotenko@gmail.com; https://orcid.org/0000-0002-3396-5461, SPIN-код: 9605-2491

А.М. Чочаева: врач-аспирант отделения офтальмоонкологии и радиологии НМИЦ «МНК «Микрохирургия глаза» им. акад. С.Н. Федорова, e-mail: chochaevaamina@mail.ru; https://orcid.org/0000-0002-0072-8275, SPIN-код: 1637-9792

В.Г. Поляков: академик РАН, д.м.н., профессор, советник директора НИИ детской онкологии и гематологии НМИЦ онкологии им. Н.Н. Блохина, заведующий кафедрой детской онкологии им. акад. Л.А. Дурнова РМАНПО, профессор кафедры оториноларингологии педиатрического факультета РНИМУ им. Н.И. Пирогова, е-mail: vgp-04@mail.ru; https://orcid.org/0000-0002-8096-0874, SPIN-код: 8606-3120

\section{Information about the authors}

A.A. Yarovoy: Dr. of Sci. (Med.), Head of Ocular Oncology and Radiology Department of the National Medical Research Center Academician S.N. Fyodorov Intersectoral Scientific and Technical Complex "Eye microsurgery", Ministry of Health of Russia, e-mail: yarovoyaa@yandex.ru; https://orcid.org/0000-0003-2219-7054, SPIN-code: 9401-4489

D.P. Volodin: Fellow Department Ocular Oncology and Radiology of the National Medical Research Center Academician S.N. Fyodorov Intersectoral Scientific and Technical Complex "Eye microsurgery", Ministry of Health of Russia, e-mail: volodin.den2016@yandex.ru; https://orcid.org/0000-0002-3660-7803, SPIN-code: 7404-9620

V.A. Yarovaya: Ophthalmologist of Ocular Oncology and Radiology Department of the National Medical Research Center Academician S.N. Fyodorov Intersectoral Scientific and Technical Complex "Eye microsurgery”, Ministry of Health of Russia, e-mail: verandreevna@gmail.com; https://orcid.org/0000-0001-8937-7450, SPIN-code: 4000-0180

T.L. Ushakova: Dr. of Sci. (Med.), Acting Head of the Pediatric Oncology Department of Surgical Treatment with Chemotherapy No. 1 (Head and Neck Tumors) of the Research Institute of Pediatric Oncology and Hematology at N.N. Blokhin National Medical Research Center of Oncology, Ministry of Health of Russia, Professor at the Department of Pediatric Oncology named after Academician L.A. Durnov at Russian Medical Academy of Continuing Professional Education, Ministry of Health of Russia, e-mail: ushtat07@mail.ru; https://orcid.org/0000-0003-3263-6655, SPIN-code: 2065-8779

E.S. Kotova: Ophthalmologist, Postgraduate Student Department of Ocular Oncology and Radiology of the National Medical Research Center Academician S.N. Fyodorov Intersectoral Scientific and Technical Complex “Eye microsurgery”, Ministry of Health of Russia, e -mail: elenkotenko@gmail.com; https://orcid.org/0000-0002-3396-5461, SPIN-code: 9605-2491

A.M. Chochaeva: Ophthalmologist, Postgraduate Student Department of Ocular Oncology and Radiology of the National Medical Research Center Academician S.N. Fyodorov Intersectoral Scientific and Technical Complex "Eye microsurgery”, Ministry of Health of Russia, e-mail: chochaevaamina@mail.ru; https://orcid.org/0000-0002-0072-8275, SPIN-code: 1637-9792

V.G. Polyakov: Academician of RAS, Dr. of Sci. (Med.), Professor, Advisor to the Director of the Research Institute of Pediatric Oncology and Hematology of N.N. Blokhin National Medical Research Center of Oncology, Ministry of Health of Russia, Head of the Pediatric Oncology Department named after Academician L.A. Durnov at Russian Medical Academy of Postgraduate Education, Ministry of Health of Russia, Professor of the Department of Otorhinolaryngology Faculty of Pediatrics at N.I. Pirogov Russian National Research Medical University, Ministry of Health of Russia, e-mail: vgp-04@mail.ru; https://orcid.org/0000-0002-8096-0874, SPIN-code: 8606-3120 


\title{
Вклад авторов
}

А.А. Яровой: разработка концепции и дизайна статьи, научное редактирование статьи

Д.П. Володин: разработка концепции и дизайна статьи, обзор публикаций по теме статьи, сбор и анализ данных, написание текста рукописи, подготовка списка литературы, составление резюме

B.А. Яровая: разработка концепции и дизайна статьи, сбор и анализ данных

Т.Л. Ушакова: научное редактирование статьи

Е.С. Котова: обзор публикаций по теме статьи, сбор и анализ данных

А.М. Чочаева: обзор публикаций по теме статьи, подготовка иллюстративного материала

В.Г. Поляков: научное редактирование статьи

\author{
Authors' contributions \\ A.A. Yarovoy: development of the concept and design of the article, scientific editing of the article \\ writing the text of the article, preparation of a list of references, composing a resume \\ V.A. Yarovaya: development of the concept and design of the article, collection and analysis of the data obtained \\ T.L. Ushakova: scientific editing of the article \\ E.S. Kotova: review of publications on the topic of the article, collection and analysis of the data obtained \\ A.M. Chochaeva: review of publications on the topic of the article, preparation of illustrative material \\ V.G. Polyakov: scientific editing of the article
}

D.P. Volodin: development of the concept and design of the article, review of publications on the topic of the article, collection and analysis of the data obtained,

Конфликт интересов. Авторы заявляют об отсутствии конфликта интересов. / Conflict of interest. The authors declare no conflict of interest.

Финансирование. Исследование проведено без спонсорской поддержки. / Funding. The study was performed without external funding.

\section{Актуальность}

Ретинобластома (РБ) - злокачественное новообразование сетчатки нейроэктодермального происхождения - является жизнеугрожающим состоянием и наиболее распространенной внутриглазной злокачественной опухолью у пациентов детского возраста [1]. РБ, как правило, встречается у детей в возрасте до 5 лет, чаще от 0 до 2 лет [2]. Показатели выживаемости в развитых странах в настоящее время приблизились к $100 \%$ [3], однако сохранение глаза и зрительных функций по-прежнему остается трудной задачей для офтальмоонкологов.

Парадигма лечения РБ на современном этапе включает проведение системной и/или локальной химиотерапии (XT) в целях хеморедукции с последующим локальным разрушением остаточной опухоли [4]. Локальные методы лечения, такие как брахитерапия (БТ), криодеструкция (КД) и транспупиллярная термотерапия (ТТТ), широко вошли в клиническую практику при недостаточной эффективности ХТ и зачастую позволяют добиться полной регрессии опухоли [5].

ТТТ является одним из ключевых методов лечения малых РБ [4, 6, 7], при этом недостаточно изучены вопросы применения этого метода в лечении опухолей на периферии глазного дна, а также в терапии остаточных опухолей после неэффективности других локальных методов.

В настоящее время количество исследований в отечественной и зарубежной литературе, посвященных ТТТ РБ, невелико, и они представлены изучением лишь отдельных аспектов данного метода лечения [6-13].

Цель исследования - оценить эффективность ТТТ при лечении детей с РБ.

\section{Материалы и методы}

В период с 2011 по 2020 г. на базе отдела офтальмоонкологии и радиологии ФГАУ «НМИЦ «МНТК
«Микрохирургия глаза» им. акад. С.Н. Федорова» Минздрава России (Москва) методом ТТТ пролечены 177 детей (224 глаза, 1156 очагов) с РБ. Из них 99 (56 \%) пациентов были мальчики, 78 (44 \%) девочки. Средний возраст детей на момент лечения составил 16,8 месяца (от 0 до 86 месяцев).

Бинокулярная форма РБ наблюдалась у $128(72,3 \%)$ пациентов (у 6 из которых отмечалось метахронное поражение), монокулярная -у49(27,7\%). В 51 (28,8 \%) случае ТТТ проведена на единственном глазу. ТТТ выполнялась на глазах, имевших РБ групп A $(n=43 ; 19 \%), \mathrm{B}(n=81 ; 36 \%), \mathrm{C}(n=31 ; 14 \%)$, $\mathrm{D}(n=63 ; 28 \%), \mathrm{E}(n=6 ; 3 \%)$.

Отягощенный семейный анамнез РБ имели $12 \%$ пациентов $(n=21), 8 \%(n=14)$ - данные генетических исследований (мутация в гене $R b 1$ ).

У $139 \quad\left(\begin{array}{ll}78 & \%\end{array}\right)$ пациентов ТТТ проводилась после выполнения системной полихимиотерапии (ПХТ), у 117 (66 \%) из них - после окончания ПХТ, у $22(14 \%)$ - в межкурсовом периоде. В 38 (22 \%) случаях ТТТ проводилась без предшествующей ПХТ, a у 14 (8 \%) пациентов (14 глаз) ТТТ использовалась как первичный метод лечения РБ.

Селективную интраартериальную ХТ (СИАХТ) (от 1 до 6 курсов на 1 глаз, в среднем 2,5 курса) перед проведением ТTТ получали 102 пациента (117 глаз); в случае наличия опухолевых отсевов в стекловидном теле у 45 пациентов (48 глаз) дополнительно применялась интравитреальная ХТ (ИВХТ) (от 1 до 12 курсов на 1 глаз, в среднем 3 курса).

При невозможности проведения и/или неэффективности системной и локальной ХТ, а также других локальных методов лечения (БТ, КД) как альтернатива энуклеации у 6 пациентов до ТТТ выполнена дистанционная лучевая терапия, у 2 пациентов - стереотаксическая радиохирургия (СТРХ).

Постэкваториально локализовались 488 (42 \%) очагов (из них 71 располагался в функционально-значимых зонах глазного дна (27 очагов - юкстапапил- 
лярно, 23 очага - в макулярной зоне, 22 очага - парамакулярно)). Преэкваториальную локализацию (на средней и крайней периферии глазного дна) имели $668(58 \%)$ очагов. Количество очагов в 1 глазу варьировало от 1 до 48 (в среднем - 5).

Средняя высота опухоли составила 1,1 мм (от 0,2 до 4,5 мм), средняя протяженность $-2,2$ мм (от 0,3 до 13,4 мм).

Всего методом ТТТ пролечены 1156 очагов РБ. Наибольший процент пролеченных очагов составили малые множественные $(n=665 ; 57 \%)$ (рис. $1-3)$ и малые одиночные $(n=375 ; 32,4 \%)$ очаги (рис. $4-6)$. В 4,4 \% случаев (51 очаг) ТТТ проводилась в случае недостаточной эффективности или рецидива после применения других локальных методов лечения (20 очагов - после БТ, 14 - после КД, 17 - после ТТТ по месту жительства). В 3,5 \% случаев (40 очагов) ТTТ использовали для разрушения венчика активной опухолевой ткани вокруг кальцинатов. При локализации очагов в функционально значимых зонах глазного дна (юкстапапиллярно, парамакулярно и в макулярной зоне) в целях сохранения зрительных функций и при высоте очагов больше 2,5 мм ТТТ применялась для лечения больших опухолей с наличием полостей, так называемой кавитарной РБ [6], что является признаком высокодифференцированной РБ $(n=25 ; 2,1 \%)$. В 21 случае использовалась методика пигмент-индуцирующей ТТТ, которая заключалась в поэтапном лазерном воздействии, как правило, по периметру опухолевого очага, при котором сформировавшиеся зоны гиперпигментации на хориоретинальном рубце после предыдущего сеанса ТТТ служат акцептором лазерного излучения при каждом последующем этапе лечения, тем самым способствуя повышению нагревания беспигментной опухоли и потенцируя эффективность ТТТ, что в конечном итоге приводит к постепенному концентрическому сокращению очага (рис. 7-9) [14].

При локализации опухоли на крайней периферии ТTТ проводилась после предварительной компрессии глазного яблока (для достижения умеренной гипотонии) и склерокомпрессии в проекции очага, что позволяло вывести опухоль в проекцию зрачка и уменьшить риск повреждения радужной оболочки [15].

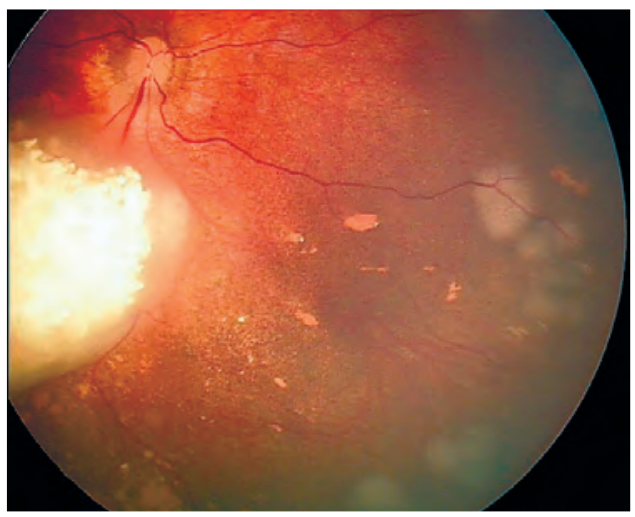

Рис. 1. Малые множественные очаги РБ до ТTТ

Fig. 1. Small multiple RB before TTT

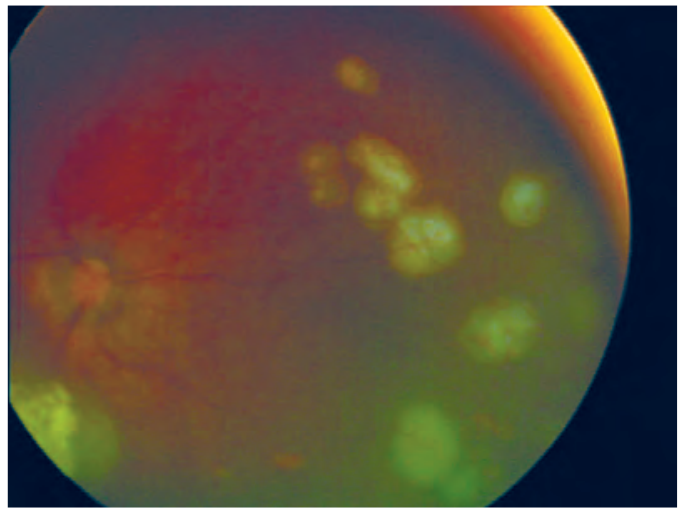

Рис. 2. Малые множественные очаги РБ через 1 мин после ТТТ Fig. 2. Small multiple RB 1 minute after TTT

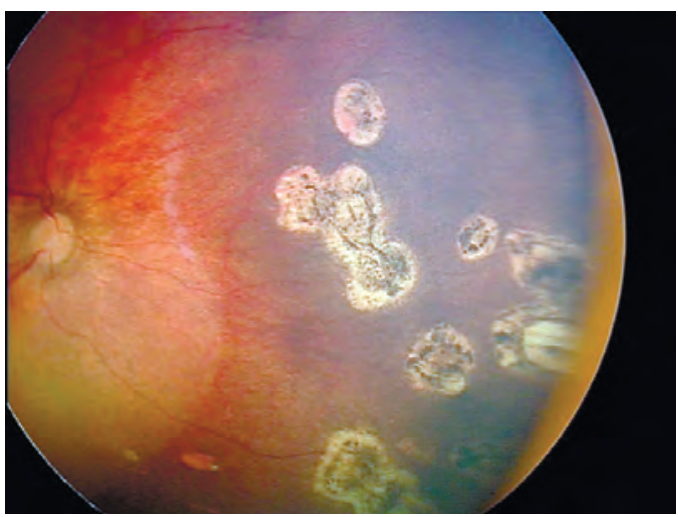

Рис. 3. Малые множественные очаги РБ через 2 мес после ТTТ

Fig. 3. Small multiple RB 2 months after TTT

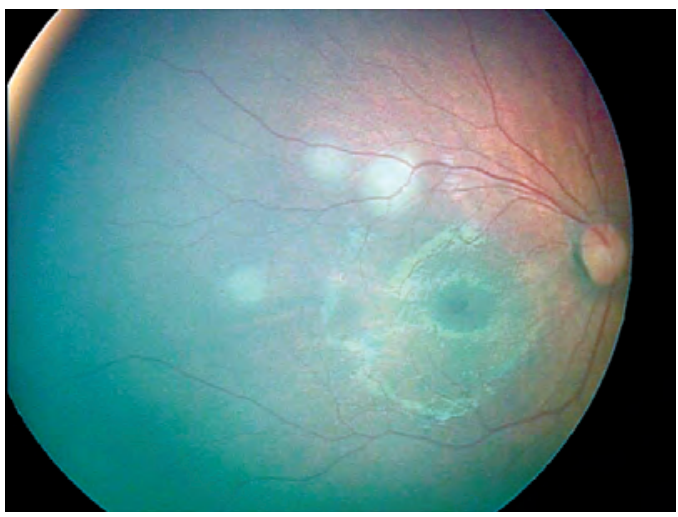

Рис. 4. Малые одиночные очаги РБ до ТТТ

Fig. 4. Small solitary RB before TTT

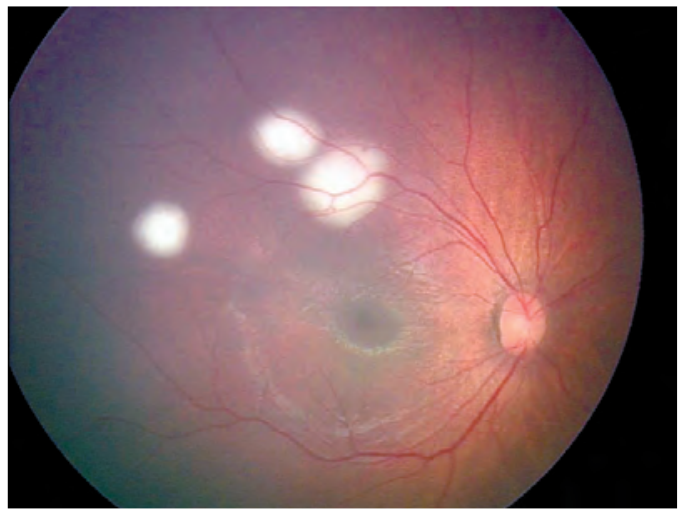

Рис. 5. Малые одиночные очаги РБ через 1 мин после ТТТ

Fig. 5. Small solitary RB 1 minute after TTT 


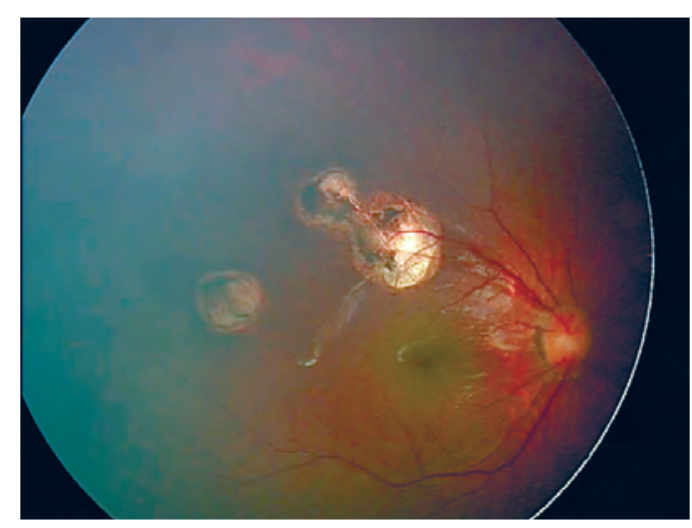

Рис. 6. Малые одиночные очаги РБ через 2 мес после ТТT

Fig. 6. Small solitary RB 2 months after TTT

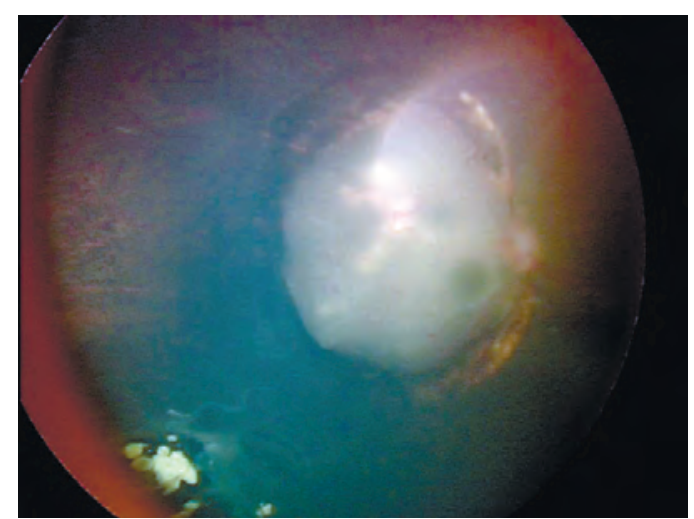

Рис. 7. Большой очаг кавитарной РБ перед проведением ТТТ Fig. 7. Large cavitary RB before TTT

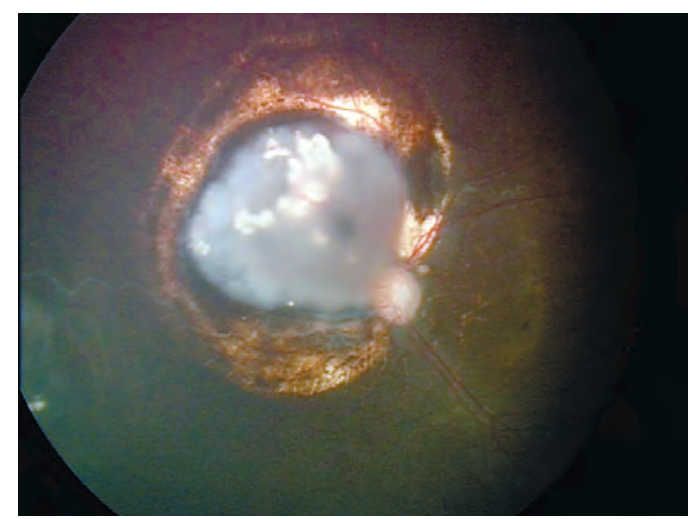

Рис. 8. Большой очаг кавитарной РБ после 2 сеансов ТТТ

Fig. 8. Large cavitary RB after 2 TTT sessions

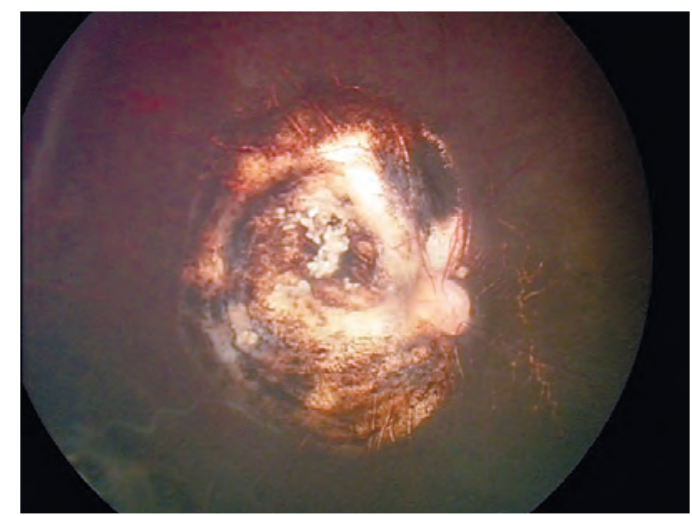

Рис. 9. Полная регрессия большого очага кавитарной РБ после 8 сеансов TTT

Fig. 9. Complete tumor regression of large cavitary RB after 8 TTT sessions
Во всех случаях лазерное вмешательство выполнялось в условиях общей анестезии после достижения максимального медикаментозного мидриаза.

TTТ проводилась с использованием диодного лазера со следующими параметрами: длина волны 810 нм, диаметр пятна - 1200 мкм, мощность от 200 до 800 мВт (средняя - 350 мВт). Экспозиция варьировала в зависимости от режима ТТТ. При аппликационном режиме экспозиция составила от 3 до 15 с, при сканирующем режиме ТТТ выполнялась с длительной непрерывной экспозицией лазерного излучения. Критерием эффективности лазерного воздействия в ходе сеанса ТTТ являлась визуализация побеления поверхности опухоли.

\section{Результаты}

Клинически полная регрессия опухоли после проведения ТТТ была достигнута в $92 \%$ случаев (1064 очага). Частичная регрессия опухоли со стабилизацией - в 0,7 \% наблюдений (8 очагов). Среднее количество сеансов ТТТ для достижения полной регрессии составило 1,7 (от 1 до 10).

Полная регрессия опухоли после 1 сеанса TTT достигнута в $54 \%$ случаев (622 очага), после 2 сеансов - в $11 \%$ (132 очага), после 3 сеансов - в $7 \%$ (85 очагов), после 4 сеансов и более- в $19 \%$ (225 очагов).

В 7 \% случаев (82 очага) в связи с продолженным ростом или рецидивом опухоли на рубце потребовалось применение других методов лечения (39 очагов - БТ, 37 - КД, 4 - БТ + КД, 1 - БТ + СТРХ, 1 - СТPX).

После проведения органосохраняющего лечения удалось сохранить 209 (93 \%) глаз. Были энуклеированы 15 (7 \%) глаз (из них в 1 случае РБ соответствовала характеристикам группы B, в 12 - группы D, в 2 - группы E), в 11 из 15 случаев энуклеация была выполнена в связи с прогрессией опухоли (из них в 4 наблюдениях энуклеация проведена в связи с продолженным ростом очага, подверженного лазерному лечению, в 7 - в связи с продолженным ростом других очагов), а в 4 случаях - в связи с развитием необратимых вторичных изменений глазного яблока (в 2 - субатрофия глазного яблока, в 2 - сочетание длительно существующей геморрагической тотальной отслойки сетчатки и тотального гемофтальма).

Один $(0,6 \%)$ из 177 пациентов умер от пинеалобластомы с генерализацией заболевания по центральной нервной системе.

Осложнения после ТТТ были представлены единичными наблюдениями: частичным гемофтальмом у 2 (1,1 \%) из 177 и претуморальным кровоизлиянием у $3(1,7 \%)$ из 177 пациентов в раннем периоде наблюдения; локальным помутнением стекловидного тела в $2(1,1 \%)$ и формированием передних синехий в $1(0,5 \%)$ из 177 случаев в отдаленном периоде наблюдения.

Средний срок наблюдения после проведения ТТТ составил 35,5 мес (от 3 до $112 \mathrm{мес).}$ 


\section{Обсуждение}

Метод ТТТ для лечения РБ был предложен J. Lagendijk в 1982 г., который он впервые применил у 2 пациентов с РБ и добился полной регрессии опухоли [16].

На сегодняшний день ТТТ играет одну из ключевых ролей в схеме комбинированного органосохраняющего лечения РБ и используется для разрушения опухолевых очагов малого размера (высотой менее 2,5 мм и протяженностью менее 3 мм), как правило, постэкваториальной локализации $[1,4]$.

Количество масштабных исследований в литератуpe, посвященных данной проблеме, невелико, описаны лишь отдельные узкие аспекты изучения данного метода [6-13, 17].

В конце 1990-х и начале 2000-х годов основными направлениями были оценка эффективности ТТТ на фоне хеморедукции опухоли после ПХТ [6], первичное использование ТTТ малых опухолей [7] и термохимиотерапия $[11,18,19]$, которая не нашла широкого применения в связи с отсутствием видимых преимуществ в виде повышения эффективности в сравнении со стандартной ТТТ.

Еще одним фактором, объясняющим небольшое количество публикаций в отечественной и зарубежной литературе, является тот факт, что ТТТ сложно оценить изолированно от других локальных методов лечения, что отмечали C. Shields et al. [6].

В 2013-2015 гг. новым направлением изучения ТTТ стало потенцирование лазерного воздействия с использованием термосенсибилизатора индоцианина зеленого, который, селективно накапливаясь в ткани беспигментной опухоли, способен усиливать поглощение лазерного излучения $[12,20]$.

В нашей работе ТTТ с контрастным усилением была проведена у 7 детей без значимого повышения эффективности лазерного воздействия.

Параметры лазерного излучения, такие как диаметр пятна, мощность и экспозиция значительно варьируют в различных исследованиях, что существенно затрудняет сравнение их результатов $[6,7,9]$.

Использованные в нашей работе параметры лазерного излучения были сопоставимы с указанными в наиболее крупных работах, посвященных данной проблеме. Так, средняя мощность в работе C. Shields et al. составила 400 мВт [6], в работе D. Abramson et al. - 420 мВт [7], а диаметр пятна варьировал от 0,8 до 2,0 мм в работе C. Shields, а в работе D. Abramson et al. составил 1,2 мм. При этом необходимо отметить более высокую продолжительность экспозиции в вышеуказанных работах (60 и 30 с соответственно) $[6,7]$.

Несмотря на значительный объем нашего исследования (177 детей (224 глаза, 1156 очагов)), эффективность ТТТ как самостоятельного метода лечения оказалась сопоставима с основными исследованиями по данной тематике. Так, в работе C. Shields et al. эффективность ТТТ составила $86 \%(n=161)$, в работе
L. Lumbroso et al. $-87 \%(n=151)$ при применении термохимиотерапии и 78 \% при использовании только TTT $(n=14)[6,11]$. В работе D. Abramson et al. эффективность ТТТ составила $92 \%(n=84)$, при этом необходимо отметить, что данное исследование было направлено на изучение эффективности ТTТ как первичного метода лечения [7]. В работе А.А. Ярового и соавт. эффективность ТТТ в лечении интраокулярной РБ составила 79,5\% $(n=116)$ [9]. В нашем исследовании клинически полная регрессия опухоли была достигнута в лечении 1064 (92\%) очагов.

Среднее количество сеансов ТТТ, необходимых для достижения полной регрессии, в нашем исследовании $(1,7 \pm 1,41$ сеанса) было также сопоставимо с другими работами (1,7 сеанса у D. Abramson et al. [7], $2-$ y L. Lumbroso et al. и C. Shields et al.) $[6,11]$.

Отдельным вопросом, определяющим эффективность ТТТ при РБ, является толщина опухоли. По данным литературы, максимальная высота опухоли при РБ, при которой возможно ее эффективное разрушение лазером, $-2-2,5$ мм [4, 6]. Ряд исследователей отмечали, что увеличение высоты опухоли уменьшает эффективность лазерного воздействия [10]. При этом сложным вопросом остается воздействие на очаги РБ, локализующиеся в функционально значимых зонах глазного дна (макулярно, парамакулярно и юкстапапиллярно), и при высоте опухоли более 2,5 мм.

Применение БТ в указанных зонах сопряжено с высоким риском развития осложнений и может приводить к значимому снижению зрительных функций [21]. Преимуществом же ТТТ является возможность прецизионного лазерного воздействия, что позволяет сохранить зрительные функции, в том числе и при локализации опухоли в вышеуказанных зонах. А предложенная нами методика пигмент-индуцированной поэтапной ТТТ позволяет добиться полной регрессии больших очагов высотой более 2,5 мм при уменьшении риска повреждения здоровых тканей в макулярной, парамакулярной и юкстапапиллярной зонах [14].

Особенностью нашего подхода к использованию ТTТ является широкое применение данного метода при лечении опухолевых очагов, расположенных преэкваториально, -58 \% $(n=668)$. По данным зарубежной литературы, ведущим методом разрушения опухолей преэкваториальной локализации высотой менее 2,5 мм является КД. По нашему мнению, при наличии единичных или множественных очагов малого размера целесообразно избегать применения КД в целях уменьшения риска развития осложнений за счет снижения травматичности и меньшей площади повреждения здоровых тканей. А проведение ТTТ с предварительной склерокомпрессией в проекции патологического очага позволяет значительно уменьшить риск ятрогенного повреждения радужной оболочки и хрусталика, а также повысить эффективность лазерного лечения опухолей на крайней периферии [15]. 


\section{Заключение}

Лазерная ТТТ является высокоэффективным методом лечения РБ и может применяться для первичных опухолей малого размера как постэкваториальной, так и преэкваториальной локализаций, остаточной опухоли при неэффективности других методов локального лечения, а также обладает эффективностью воздействия на очаги кавитарной РБ, расположенных в функционально значимых зонах глазного дна. При этом метод ТТТ является безопасным, с единичными осложнениями и не представляет угрозы для глаза, а прецизионность лазерного воздействия делает возможным не только достижение полной регрессии опухоли, но и сохранение зрительных функций.

\section{J И TEPAT V PA / REFERENBES}

1. Rao R., Honavar S.G. Retinoblastoma. Indian J Pediatr 2017;84(12):937-44. doi: 10.1007/s12098-017-2395-0.

2. Ушакова Т.Л. Этиология, патогенез, клиника, диагностика ретинобластомы. Проблемы органосохраняющего лечения. Детская онкология 2003;1:40-5. [Ushakova T.L. Etiology, pathogenesis, clinical picture, diagnosis of retinoblastoma. Problems of organpreserving treatment. Detskaya onkologiya $=$ Pediatric Oncology 2003;1:40-5. (In Russ.)].

3. Fabian I.D., Abdallah E., Abdullahi S.U., Abdulqader R.A., Boubacar S.A., Ademola-Popoola D.S., Adio A., Afshar A.R., Aggarwal P., Aghaji A.E., Ahmad A. Global Retinoblastoma Presentation and Analysis by National Income Level. JAMA Oncol 2020;6(5):685-95. doi: 10.1001/jamaoncol.2019.6716.

4. Ancona-Lezama D., Dalvin L.A., Shields C.L. Modern treatment of retinoblastoma: A 2020 review. Indian J Ophthalmol 2020;68(11):2356-65. doi: 10.4103/ijo.IJO_721_20.

5. Ушакова Т.Л., Трофимов И.А., Горовцова О.В., Яровой А.А., Саакян С.В., Летягин И.А., Матинян Н.В., Кукушкин А.В., Мартынов Л.А., Погребняков И.В., Иванова О.А., Серов Ю.А., Яровая В.А., Глеков И.В., Виршке Э.Р., Долгушин Б.И., Поляков В.Г. Новая эра органосохраняющего лечения детей с интраокулярной ретинобластомой в России: мультицентровое когортное исследование. Онкопедиатрия 2018;5(1):51-69. doi: 10.15690/onco.v5i1.1866. [Ushakova T.L., Trofimov I.A., Gorovtsova O.V., Yarovoy A.A., Saakyan S.V., Letyagin I.A., Matinyan N.V., Kukushkin A.V., Martynov L.A., Pogrebnyakov I.V., Ivanova O.A., Serov Y.A., Yarovaya V.A., Glekov I.V., Virshke E.R., Dolgushin B.I., Polyakov V.G. A New Era of Organ-Preserving Treatment in Pediatric Intraocular Retinoblastoma in Russia: A Multicentre Study. Onkopediatria = Oncopediatrics 2018;5(1):51-69. (In Russ.)].

6. Shields C.L., Santos M.C.M., Diniz W., Gündüz K., Mercado G., Cater J.R., Shields J.A. Thermotherapy for Retinoblastoma. Arch Ophthalmol 1999;117(7):885-93. doi: 10.1001/archopht.117.7.885.

7. Abramson D.H., Schefler A.C. Transpupillary thermotherapy as initial treatment for small intraocular retinoblastoma. Ophthalmology 2004;111(5):984-91. doi: 10.1016/j.ophtha.2003.08.035.

8. Кривовяз О.С., Булгакова Е.С., Яровой А.А. Способ потенцирования транспупиллярной лазерной термотерапии при ретинобластоме. Современные технологии в офтальмологии 2015;3:91-3. [Krivovyaz O.S., Bulgakova E.S., Yarovoy A.A. A method of potentiating transpupillary laser thermotherapy for retinoblastoma. Sovremennye tekhnologii v oftal'mologii = Modern Technologies in Ophthalmology 2015;3:91-3. (In Russ.)].

9. Яровой А.А., Ушакова Т.Л., Поляков В.Г., Кривовяз О.С., Горовцова О.В. Транспупиллярная диод-лазерная термотерапия в схеме органосохраняющего лечения интраокулярной ретинобластомы у детей. Х Съезд офтальмологов России - 2015. С. 218. [Yarovoy A.A., Ushakova T.L., Polyakov V.G., Krivovyaz O.S., Gorovtsova O.V. Transpupillary diode-laser thermotherapy in the regimen of organ-preserving treatment of intraocular retinoblastoma in children. X Congress of Ophthalmologists of Russia - 2015. P. 218. (In Russ.)].

10. Саакян С.В., Тацков Р.А., Мякошина Е.Б., Ушакова Т.Л., Поляков В.Г. Эффективность транспупиллярной термотерапии в комбинированном лечении малых кальцифицированных ретинобластом. Российский офтальмологический журнал 2017;10(3):71-7. doi: 10.21516/2072-0076-2017-10-3-71-77. [Saakyan S.V., Tatskov R.A., Myakoshina E.B., Ushakova T.L., Polyakov V.G.
Transpupillary thermotherapy efficiency in the combined treatment of small calcified retinoblastoma. Rossiyskiy oftal'mologicheskiy zhurnal = Russian Ophthalmological Journal 2017;10(3):71-7. (In Russ.)].

11. Lumbroso L., Doz F., Levy C., Dendale R., Vedrenne J., Bours D., Zucker J.M., Asselain B., Desjardins L. Diode laser thermotherapy and chemothermotherapy in the treatment of retinoblastoma. J Fr Ophtalmol 2003;26(2):154-9. PMID: 12660589.

12. Hasanreisoglu M., Saktanasate J., Schwendeman R., Shields J.A., Shields C.L. Indocyanine Green-Enhanced Transpupillary Thermotherapy for Retinoblastoma: Analysis of 42 Tumors. J Pediatr Ophthalmol Strabismus 2015;52(6):348-54. doi: 10.3928/01913913-20150929-17.

13. Яровой А.А., Ушакова Т.Л., Поляков В.Г., Булгакова Е.С., Кривовяз О.С., Горовцова О.В. Результаты локального лечения ретинобластомы при недостаточной эффективности полихимиотерапии. Офтальмохирургия 2014;(1):79-84. [Yarovoy A.A., Ushakova T.L., Polyakov V.G., Bulgakova E.S., Krivovyaz O.S., Gorovtsova O.V. Results of local treatment of retinoblastoma after polychemotherapy. Oftal'mokhirurgiya = Fyodorov Journal of Ophthalmic Surgery 2014;(1):79-84. (In Russ.)].

14. Яровой А.А., Кривовяз О.С. Способ лазерного лечения резистентных форм ретинобластомы у детей. Патент на изобретение № RU 2600145 C1, 2016 г. [Yarovoy A.A., Krivovyaz O.S. Method for laser treatment of resistant forms of retinoblastoma in children. Patent for invention No. RU 2600145 C1, 2016 (In Russ.)].

15. Яровой А.А., Дога А.В., Логинов Р.А., Яровая В.А., Котельникова А.В. Способ лазерного лечения патологии крайней периферии глазного дна при обратной офтальмоскопии. Патент на изобретение № RU 2715194 C1, 2020 г. [Yarovoy A.A., Doga A.V., Loginov R.A., Yarovaya V.A., Kotel'nikova A.V. Method for laser treatment of pathology of the extreme periphery of the fundus during reverse ophthalmoscopy. Patent for invention No. RU 2715194 C1, 2020. (In Russ.)].

16. Lagendijk J.J. A microwave heating technique for the hyperthermic treatment of tumours in the eye, especially retinoblastoma. Phys Med Biol 1982;27(11):1313-24. doi: 10.1088/0031-9155/27/11/002.

17. Desjardins L., Chefchaouni M.C., Lumbroso L., Levy C., Asselain B., Bours D., Vedrenne J., Zucker J.M., Doz F. Functional results after treatment of retinoblastoma. J Am Assoc Pediatr Ophthalmol Strabismus 2002;6(2):108-11. doi: 10.1067/mpa.2002.121451.

18. Levy C., Doz F., Quintana E., Pacquement H., Michon J., Schlienger P., Validire P., Asselain B., Desjardins L., Zucker J.M. Role of chemotherapy alone or in combination with hyperthermia in the primary treatment of intraocular retinoblastoma: preliminary results. Br J Ophthalmol 1998;82(10):1154-8. doi: 10.1136/bjo.82.10.1154.

19. Schueler A.O., Jurklies C., Heimann H., Wieland R., Havers W., Bornfeld N. Thermochemotherapy in hereditary retinoblastoma. Br J Ophthalmol 2003;87(1):90-5. doi: 10.1136/bjo.87.1.90.

20. Francis J.H., Abramson D.H., Brodie S.E., Marr B.P. Indocyanine green enhanced transpupillary thermotherapy in combination with ophthalmic artery chemosurgery for retinoblastoma. Br J Ophthalmol 2013;97(2):164-8. doi: 10.1136/bjophthalmol-2012-302495.

21. Schueler A.O., Flühs D., Anastassiou G., Jurklies C., Sauerwein W., Bornfeld N. Beta-Ray Brachytherapy of Retinoblastoma: Feasibility of a New Small-Sized Ruthenium-106 Plaque. Ophthalmic Res 2006;38(1):8-12. doi: 10.1159/000088259. 